\title{
OPTIMIZATION OF LOADING FACILITIES AT THE TERMINAL
}

\author{
Danutė Bagdonienè \\ Dept of Mathematical Statistics, Vilnius Gediminas Technical University, \\ Sauletekio al. 11, LT-10223 Vilnius, Lithuania. E-mail: mstk@fm.vgtu.lt
}

Received 17 June 2007; accepted 1 February 2008

\begin{abstract}
The problems of stock management and proper choice of transport facilities are defined and analysed taking into account the relationship between cost of transportation and vehicle's capacity and size of cargo lots. By using mathematical statistical methods, the optimal vehicle's capacity for a particular lot on the routes for taking the cargo out as well as the periodicity of cargo delivery are determined.
\end{abstract}

Keywords: cost of transportation, vehicle's capacity, stock management.

\section{Introduction}

In the present paper, major functions of enterprises engaged in freight transportation by road which is associated with taking cargoes in and out from the terminal as well as the ways of coordinating the work of loading and transport facilities have been analysed and simulated. The time of harmonized operation of transport and loading facilities may be specified for vehicles which are running or being loaded or uploaded. The delay time of a vehicle due to the operations of loading and unloading consists of the transportation processes taking a long time, i.e. loading and unloading, manoeuvring when loading is over, manoeuvring over the area of cargo transfer, lifting the vehicle's sides, fixing the cargo, documentation, etc. The highest standard delay time due to loading and unloading operations is determined by transportation tariffs depending on the methods of cargo loading and unloading as well as on the types of hoisting and loading mechanisms and the shape of cargo. The delay time of a vehicle caused by loading and unloading operations depends on many factors, therefore it may be treated as a random value.

A process of transportation may be described in terms of mass service network performance, the service stages of which are assumed to be a sequence of loading/ unloading operations as well as the movement of a loaded or empty vehicle on particular route sections (Baublys and Petrauskas 2002).

The operational characteristics of mass service may be determined based on various criteria (Bagdoniene and Mazūra 2004). Major of them are the total costs of loading/unloading one ton of cargo or of one vehicle's loading/unloading operation and relative operation efficiency in a system (at a loading/ unloading terminal).
For the sake of simplicity of calculation, only the costs relating to losses as part of the total costs characterizing the efficiency of operation may be considered.

\section{A complex solution to the problems of transport facilities selection and stock management}

Taking into account the dependence of transportation cost on vehicle's capacity and size, models of stock management and transport facilities selection may be analysed from various perspectives.

The cost of freight transportation varies depending on particular conditions.

When the amount of a particular lot of goods $g$ exceeds vehicle's capacity $\left(g>q \gamma_{s t}\right)$, cost of transportation does not depend on the amount of goods in a lot, implying that, in this case, the delivery cost of 1 ton of cargo is constant:

$$
S_{T}^{(1)}(g)=a_{1} ; g>q \gamma_{s t} .
$$

If the lot size corresponds to the capacity of a vehicle chosen from a number of vehicles in a queue $\left(g_{d}=q \gamma_{s t}\right)$, the delivery cost of 1 ton of goods may be obtained from the formula expressing cost of freight transportation on the delivery routes, i.e., when $\bar{h}_{(i-1)-i}=0$ and $q \gamma_{s t}=g_{d}$ $\left(q \gamma_{s t}\right.$ is a coefficient expressing the use of vehicle's capacity determining its work load). In this case, the following function describes the relationship between the delivery cost of 1 ton of cargo and a lot of goods:

$$
S_{T}^{(2)}(g)=a_{2}+\frac{b_{2}}{g}+c_{2} g ; g=q \gamma_{s t} .
$$

The delivery cost of 1 ton of cargo on the routes $g<$ $q \gamma_{s t}$ depending on the average lot size is obtained from the following formula: 


$$
S_{T}^{(3)}(g)=a_{3}+\frac{b_{3}}{g} ; g<q \gamma_{s t} .
$$

The costs of cargo storage consist of storage costs, natural loss of weight and losses due to deterioration of consumer goods and costs of goods 'freezing. When containers are used for cargo delivery, the cost of their removal from the whole cycle should be taken into account.

The cost of the storage of goods $x$ per unit of time is as follows:

$$
S_{g o}(x)=x C_{g o}+C_{f} g_{t} k_{d e l},
$$

where $C_{g o}, C_{f}$ - the costs of storing 1 ton of cargo and containers per unit of time; $g_{t}$ - mass of containers in one cargo delivery, $\mathrm{t} ; k_{d e l}=t_{f} / t_{g o}$ - coefficient of container removal periodicity ( $t_{f}$ and $t_{g o}$ - time intervals between taking out of containers and cargo delivery).

Since

$$
g=g_{t}+g_{p}=\left(1+\frac{1}{k_{t}}\right) g_{t},
$$

where $g_{p}$ is mass of goods in one delivery operation, $t$; $k_{t}=g_{t} / g_{p}-$ container coefficient, then:

$$
S_{g o}(x)=x C_{g o}+\frac{C_{f} k_{d e l} k_{t}}{1+k_{t}} g \text {. }
$$

The above model of managing stocks of goods when the costs of handling do not depend on the size of lots is simple. However, the following case describing the cost of handling a lot of goods $g$ is more common:

$$
S_{3}(g)=a_{h}+b_{h} g
$$

where $a_{h}$ and $b_{h}$ are constant values.

Given the above relationships, a model of costs may be expressed as follows:

- The cost of making an order for a lot of goods:

$$
S_{3}(g)=\left\{\begin{array}{l}
a_{h}+b_{h} g, g>0 \\
0, g=0
\end{array}\right.
$$

- The cost of delivering 1 ton of cargo for a lot of goods $g$ is:

$$
S_{T}(g)=\left\{\begin{array}{l}
a_{1}, g>q \gamma_{s t} ; \\
a_{2}+\frac{b_{2}}{g}+c_{2} g, g=q \gamma_{s t} \\
a_{3}+\frac{b_{3}}{g}, g<q \gamma_{s t} .
\end{array}\right.
$$

- The cost of stock storage $x$ per unit of time is as follows:

$$
S_{g o}(x)=\left\{\begin{array}{l}
C_{g o} x+p g, x \geq 0 ; \\
p g, x<0 .
\end{array}\right.
$$

Let us consider the simplest management model of storing the same goods for a given constant demand with the intensity $r$. The delivery value $g$ connected with the relationship $g_{p}=g /\left(1+k_{t}\right)$ of the delivered lot of goods is un- known. When the demand and supply are known, there is no need for piling up goods, because a new order is made when the stock of goods is over. The function $x(t)=g_{p}-r t$ indicates the available stock of goods at the time interval $t$.

The dynamic problem is solved individually by considering the sequence of statistical problems for any interval of delivery $t_{d e l}=g_{p} / r$ specified by the conditions $x\left(t_{d e l}\right)=0$. An average stock value during a particular period of time is as follows:

$$
\bar{x}=\frac{1}{t_{d e l}} \int_{0}^{t_{d e l}} x(t) d t=\frac{g_{p}}{2}=\frac{g}{2\left(1+k_{t}\right)} .
$$

The relationship of the total costs calculated for one ton of cargo depending on the lot size is expressed in the following way:

$$
S(g)=\frac{S_{3}(g)}{g}+S_{T}(g)+\frac{S_{g o}(\bar{x}) t_{d e l}}{g} .
$$

The considered alternatives of cargo delivery vary in the delivery cost $S_{T}(g)$. However, in any case, the relationship between the total costs and the size of cargo lot is described by the same formula:

$$
S(g)=a+\frac{b}{g}+c g,
$$

where only the coefficients $a, b, c$ (Table) vary.

Constant coefficients for determining the total costs by the formula (13)

\begin{tabular}{cccc}
\hline $\begin{array}{c}\text { Delivery } \\
\text { variant }\end{array}$ & \multicolumn{3}{c}{ Coefficient } \\
\cline { 2 - 4 }$g>q \gamma_{s t}$ & $a_{1}+a_{h}$ & $a_{h}$ & $\frac{0,5 C_{g o}+C_{f} k_{d e l} k_{t}}{\left(1+k_{t}\right)^{2} r}$ \\
\hline$g=q \gamma_{s t}$ & $a_{2}+b_{h}$ & $a_{h}+b_{2}$ & $\frac{0,5 C_{g o}+C_{f} k_{d e l} k_{t}}{\left(1+k_{t}\right)^{2} r}+c_{2}$ \\
\hline$g<q \gamma_{s t}$ & $a_{3}+b_{h}$ & $a_{h}+b_{3}$ & $\frac{0,5 C_{g o}+C_{f} k_{d e l} k_{t}}{\left(1+k_{t}\right)^{2} r}$ \\
\hline
\end{tabular}

Based on the expression $\delta S(g) / \delta g$ we can find the optimal lot size:

$$
g_{o p t}=\sqrt{\frac{b}{c}} .
$$
follows:

The most optimal periodicity of load delivery is as

$$
t_{\text {opt.del }}=\frac{g_{p . o p t}}{r}=\frac{1}{\left(1+k_{t}\right) r} \sqrt{\frac{b}{c}} .
$$

Let us assume that in the first delivery option $(g>$ $q \gamma_{s t}$ ) the cargo is not delivered in containers, i.e. $k_{t}=0$, then we will obtain previously discussed Wilson's formula:

$$
g_{o p t}=\sqrt{\frac{2 C_{o b} r}{C_{g o}}} .
$$


where $C_{o b}$ - the observance of delivery terms. In the first model, only the cost of delivery and storage are taken into account. The delivery of small lots of goods at short intervals is more expensive from the organizational point of view, while the delivery of big lots at long intervals increases storage costs.

The third model $\left(g>q \gamma_{s t}=q \gamma_{o}\right)$ takes into account the cost of delivery depending on the lot size, however, only on the routes where cargo is taken out. In this case, the formula (14) determines the optimal average size of the cargo lot for several customers on the same route, while the lot size for individual customers should be determined depending on demand.

The coefficient $c$ depends on the same factors as those relating to the first model, while the value of coefficient $b$ is higher than that of $b_{3}$ depending on the average distance run by a vehicle between the two intermediate closest points $\bar{l}_{(i-1)-i}$, zero vehicle's run $l_{n}$, and additional delay time of a vehicle at every intermediate point $t_{3}$.

Since choosing the proper vehicle capacity for freight delivery on the dispatching routes is an extreme problem and the optimal vehicle's capacity depends on the average lot size, the two problems including the choice of the lot size and vehicle's capacity are solved together.

The average lot size and vehicle's capacity are found from the following system of equalities:

$$
\left.\begin{array}{l}
\frac{\delta S\left(g_{d}, q \gamma_{o}\right)}{\delta g_{d}}=0 ; \\
\frac{\delta S\left(g_{d}, q \gamma_{o}\right)}{\delta\left(q \gamma_{o}\right)}=0 ;
\end{array}\right\} g_{d}=g .
$$

The relationship between the total cost for 1 ton of cargo and the average lot size $g$ is expressed by the equality (13) obtained by substituting the function $S_{T}^{(3)}$ taken from the formula (3) into the formula (12).

By differentiating the equality expressing the total cost of transporting 1 ton of cargo according to $g_{d}$ and $q \gamma_{i s}$ and assuming the results to be equal to zero, we obtain the systems (18) and (19). One of them is as follows:

$$
\sqrt{\frac{g_{d}=\left(1+k_{t}\right) \times}{\left[a_{h}\left(1+k_{l}\right)+\frac{C_{k m}}{\delta}\left(\bar{l}_{i-1)-i}+\frac{l_{n}}{T_{d}} t_{h}\right)+C_{f} t_{h}\right]}}
$$

where $C_{k m}$ - costs of $1 \mathrm{~km}$ run of a vehicle, $k_{l}$ - coefficient taking into account additionally taken cargo, $T_{d}$ - time of vehicle's operation, $t_{t}$ - time of unloading the unit cargo mass. The second formula is expressed in the following way:

$$
\sqrt{\frac{g_{d}\left(2 \bar{l}_{i}-\bar{l}_{(i-1)-i}\right) a_{k m}}{b_{k m} \bar{l}_{(i-1)-i}+\left(\frac{l_{n}}{T_{d}} b_{k m}+\delta b_{f}\right)\left[t_{t}\left(1+k_{l}\right) g_{d}+t_{h}\right]}}
$$

The values $C_{k m}$ and $C_{f}$ in the above formula depend on vehicle's capacity. The expression (19) determines the optimal capacity of vehicles operating on the routes for taking the cargo out. The system of the above equalities may be solved in a simple way by determining vehicle's capacity and choosing an appropriate size of a lot of goods. Then, the optimal capacity may be determined for the vehicle. The calculations are made until the same results are obtained from two consecutive calculations, i.e. they yield the same value of vehicle's capacity. The latter and the corresponding average size of a lot of goods will be considered optimal.

\section{Conclusions}

The problems of stock management and proper choice of transport facilities are defined and analysed taking into account the relationship between cost of transportation and vehicle's capacity and size of cargo lots. By using mathematical statistical methods, the optimal vehicle's capacity for a particular lot on the routes for taking the cargo out as well as the periodicity of cargo delivery are determined.

\section{References}

Bagdoniené, D.; Mazūra, S. 2004. Optimization of technological processes at the terminal, Transport 19(4): 149-156.

Baublys, A.; Petrauskas, B. 2002. Transporto terminalai [Transport terminals]. Vilnius: Technika. 285 p. 